\title{
Capacitance-Voltage Characteristics of InAs Dots: A Simple Model
}

\author{
A. J. Chiquito, Yu. A. Pusep, S. Mergulhão ${ }^{1}$, and J. C. Galzerani ${ }^{1}$ \\ Instituto de Fisica de São Carlos, Universidade de São Paulo, \\ C.P. 639, 13650-970, São Carlos, São Paulo, Brazil \\ ${ }^{1}$ Departamento de Fisica, Universidade Federal de São Carlos, \\ C.P. 676, 13565-905, São Carlos, São Paulo, Brazil
}

Received on 19 October, 2001. Revised version received on 18 April, 2002.

\begin{abstract}
An electrostatic model was presented for the calculation of the capacitance-voltage characteristics of a semiconductor structure where quantum dots were embedded. The model was based on the linear coupling between the contributions of the quantum dots and the bulk host. We further applied this model to an InAs/GaAs self-assembled quantum dots system. The calculated capacitance was found in good agreement with the experimental curves, providing parameters of the dots ensemble, as the excitation energy of the confined electrons.
\end{abstract}

\section{Introduction}

Self-assembled quantum (SAQDs) dots producing zerodimensional electron confinement are usually obtained growing materials with different lattice parameters which provides high quality structures. In this work we study the InAs/GaAs system in which the $7 \%$ lattice mismatch between the epitaxial film (InAs) and the GaAs substrate induces the Stranski-Krastanow growth mode and then, 3D InAs islands are formed [1]. In order to investigate the electronic and structural properties of the dots, electrical methods for characterization such as the admittance spectroscopy, deep level transient spectroscopy and the capacitance-voltage $(\mathrm{C}-\mathrm{V})$ technique are typically used $[2,3,4,5]$.

The energetic distribution of electrons in a system implicitly includes the physical properties of the system and it depends essentially on the local density of states (DOS). In this way, the capacitance-voltage (energy) relationship, measuring the number of charges as a function of the energy is an useful technique to study the properties of the confined electrons in quantum dots as well in other low dimensional semiconductor systems. Note that the system dimensionality is naturally included in the capacitance-DOS relationship. The work here described deals with the role of the electrical coupling between the quantum dots and the bulk contributions and their influence on the capacitance.

\section{Samples and experimental methods}

The structures of the samples [Fig. 1(a)] used in this study were based on a field effect device[6]. The samples were grown by molecular-beam epitaxy on (100) GaAs highly doped substrates. The growth process of the structures can be summarized as follows: after an oxide desorption, a GaAs:Si buffer layer was grown at $\mathrm{T}=580^{\circ} \mathrm{C}$ followed by a doped $(\mathrm{GaAs})_{17} /(\mathrm{AlAs})_{2}: \mathrm{Si}$ smoothing superlattice and a $25 \mathrm{~nm}$ thick undoped GaAs layer. The next stage was the formation of the InAs dots. The temperature was reduced to $500^{\circ} \mathrm{C}$ (in order to minimize the process of In segregation) and an InAs layer with the nominal thickness 2.3 ML was grown. The formation of the dots was observed after the deposition of $1.8 \mathrm{ML}$ thick InAs. The temperature was increased to $\mathrm{T}=580^{\circ} \mathrm{C}$ and an undoped GaAs layer $(25 \mathrm{~nm})$ was grown. At the top of the structures undoped $(\mathrm{GaAs})_{11} /(\mathrm{AlAs})_{4}$ (with total thicknesses 120 $\mathrm{nm}$ ) superlattices were grown in order to increase the impedance of the samples and then, allow the voltage range applied to the samples to be extended. Finally, in order to prevent surface oxidation in air, the structures were capped with $5 \mathrm{~nm}$ GaAs layers. During the growth, the InAs and GaAs fluxes were fixed at 0.1 and 0.5 respectively, while $\mathrm{P}_{\mathrm{AS}}=5 \times 10^{-6} \mathrm{~Pa}$.

For the Schottky diode construction, a conventional ohmic contact was fabricated to the substrate, with an AuGeNi alloy annealed at $450^{\circ} \mathrm{C}$ for $120 \mathrm{~s}$; the Schottky contact was formed by the deposition of a $100 \mathrm{~nm}$ gold

\footnotetext{
*Corresponding author: chiquito@df.ufscar.br. Also at Dept. de Física, Universidade Federal de São Carlos, São Carlos, São Paulo.
} 
layer with $500 \mu \mathrm{m}$ diameter. The admittance measurements were carried out using a standard lock-in technique (using a SR530 Stanford dual phase lock-in amplifier) with a $10 \mathrm{mV}$ measuring signal $(\mathrm{f}=100 \mathrm{kHz})$. The samples were mounted in cryostat coupled with the measurement system and the $\mathrm{C}-\mathrm{V}$ curves were measured at $\mathrm{T}=10 \mathrm{~K}$.

\section{Model}

Consider the sketch of the energy band diagram depicted in Fig. 1(b). Generally, the capacitance of the whole structure is an unknown function $(F)$ of the applied voltage:

$$
C=F\left[\frac{d Q_{B u l k}}{d V}, \sum_{i} \frac{d Q_{S A Q D s}^{i}}{d V}\right]
$$

where $Q_{B u l k}$ and $Q_{S A Q D s}^{i}$ are the charges of the bulk and those confined in the discrete levels of the SAQDs (summation is assumed over all occupied states). In order to calculate the capacitance one needs to take into account the energy dependence of all quantized states using the Schrödinger's and Poisson's equations in a self-consistent procedure [7].

However, we can use a particular form for the function $F$ based on some considerations: we can suppose that the plane containing the dots acts like an equipotential surface. Considering only the ground state of the occupied dots one may assimilate that the contribution of the electrons in the SAQDs region will be a small correction to the depletion layer capacitance. Due to the proximity between the plane containing the SAQDs and the highly doped buffer we consider that these two layers are near the electrostatic equilibrium [8]. Using this approximation we are describing the system as a pure electrostatic system, where the quantum effects are accounted by the density of states.

The capacitance in a Schottky device is directly related with the charge inside the depletion region, which in this case is given by:

$$
\begin{aligned}
Q_{\text {Depletion }} & =Q_{\text {Bulk }}+Q_{S A Q D s} \\
& =q S\left(N_{D} w-n_{S A Q D s}\right)
\end{aligned}
$$

where $\mathrm{S}$ is the Schottky contact area, $N_{D}$ is the bulk doping level, $w$ is the width of the depletion region and $n_{S A Q D s}$ is the number of electrons in the plane containing the dots.

Applying a voltage at the Schottky contact and solving the Poisson's equation for this voltage we can calculate the electric potential along the structure. Using $t \simeq w$ due to the low doping level of the samples (undoped) we obtain

$$
V=t \frac{Q_{B u l k}}{\varepsilon_{s}}+\frac{t}{\varepsilon_{s}}\left(1-\frac{t_{2}}{t}\right) Q_{S A Q D s},
$$

where $\varepsilon_{s}$ is the dielectric constant of GaAs and $t, t_{2}$ are defined on Fig. 1(b). Equation (3) gives a general [9] expression for the electric potential in a structure with an accumulation layer (low dimensional system) inside the depletion region and it can be only used when there is no dc current flowing in the sample. The fraction $\frac{t_{2}}{t}$ represents the component of the applied voltage at the plane containing the SAQDs when no band bending is present; obviously, this is an approximation because the presence of charges inside the dots always induces some band bending that was neglected.

By differentiation of eqs. (2) and (3) with respect to the applied voltage we obtain $\mathrm{dQ}$ and $\mathrm{dV}$. After some algebra and using the definition $C=\frac{d Q}{d V}$, the capacitance of the whole structure was obtained as follows:

$$
C=C_{B u l k}+C_{S A Q D s},
$$

where $\left[\Phi_{B}\right.$ is the Schottky barrier height, Fig. 1(b)]

$$
\begin{gathered}
C_{B u l k}=\sqrt{\frac{q N_{D} \varepsilon_{s}}{2\left(\Phi_{B}-V\right)}}, \\
C_{S A Q D s}=q \frac{t_{2}}{t} \frac{d}{d V}\left[\int_{0}^{\infty} D(\varepsilon, V) f(\varepsilon, V) \varepsilon\right],
\end{gathered}
$$

where

$D(\varepsilon, V)=\frac{N_{S A Q D s}}{\sqrt{\frac{\pi}{2} \Delta \varepsilon}} \exp \left[-2\left(\frac{\varepsilon+\varepsilon_{S A Q D s}+q \frac{t_{2}}{t} V}{\Delta \varepsilon}\right)^{2}\right]$

(a)

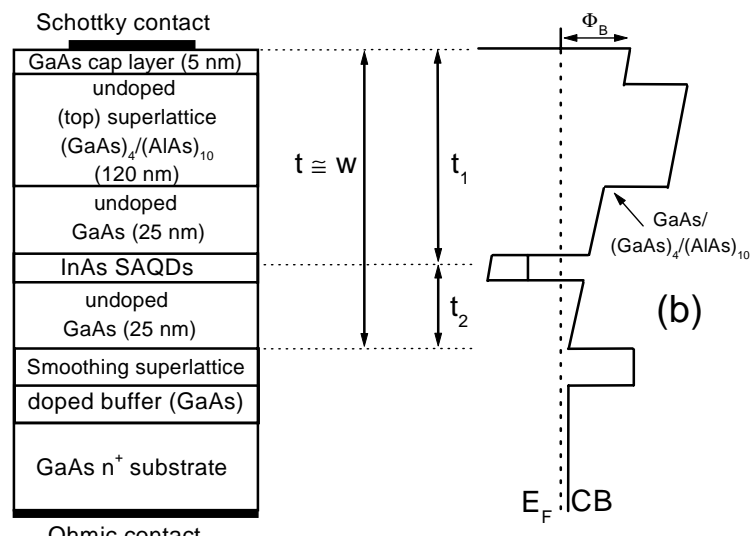

Figure 1. Panel (a) shows a sketch of the samples and in panel (b) is shown the corresponding sketch of the energy band diagram. $\Phi_{B}$ is the Schottky barrier height, $E_{F}$ is the Fermi energy.

In the above equations, $D(\varepsilon, V)$ is the electron density of states, $f(\varepsilon, V)$ is the Fermi-Dirac energy distribution, $\frac{t_{2}}{t} V$ the voltage across the quantum dots. The local density of states in the InAs SAQDs can be written in a delta function form in the ideal case (no dots size dispersion); here, we should include the effects of the dots size dispersion by taking into account 
a gaussian broadening of the density of states [10] with a characteristic energy dispersion $\Delta \varepsilon$. $\varepsilon_{S A Q D s}$ is the excitation energy of the trapped electrons in SAQDs. Notice that $C_{S A Q D s}$ also takes into account the effects of the temperature which are included into the FermiDirac distribution.
As above mentioned and in accordance with eqs. (4) and (6) capacitance measurements are well suited for investigations on the density of states and on the system dimensionality. This situation is directly observed in the low temperature limit $(T \rightarrow 0)$ where $C_{S A Q D s}$ is well described by $[11,12]$

$$
C_{S A Q D s}=\frac{t_{2}}{t} q^{2}\left[D\left(\varepsilon_{F}\right)+\frac{\pi^{2}}{6}(k T)^{2} \frac{\partial^{2}}{\partial \varepsilon_{F}^{2}} D\left(\varepsilon_{F}\right)+\frac{7 \pi^{4}}{360}(k T)^{4} \frac{\partial^{4}}{\partial \varepsilon_{F}^{4}} D\left(\varepsilon_{F}\right)+\ldots\right],
$$

where $\varepsilon_{F}$ is the Fermi energy. In this case, the tunneling is the dominant mechanism of the charging/discharging process of the electron states in the SAQDs. Under the above simplifying conditions (no dc current flowing in the structure and no band bending) the capacitance is a direct method of measuring the DOS[12]. Considering only the first term of the expansion in eq. (8) in powers of $\mathrm{kT}$, we are able to use the DOS-capacitance relantionship in order to identificate the dimensionality of an electron system. However, eq. (8) should be carefully used (in a qualitative approach) because in an interating system (as the SAQDs) the DOS depends on the number of electrons in the system.

The electrical coupling established in eq. (4) also reflects the incomplete screening of the electric field by all parts of the structure. The nonabrupt edge of the depletion region allows a small tail of the field to penetrate through the first barrier [width $t_{1}$, Fig. 1(b)] into the SAQDs plane. Thus, when the electric field is depleting the first barrier, the SAQDs region is also contributing to the capacitance. The distribution of electrons in the plane of the SAQDs will not screen entirely the field which penetrates into the second barrier [width $t_{2}$, Fig. 1(b)]. Then at a given voltage applied to the Schottky contact all parts of the structure are contributing to the measured capacitance. Certainly, the electrical coupling between the barriers and quantum dots depends on the interlayer distances and on the doping level of these regions.

\section{Results and discussion}

Next, we applied the model to fit the experimental $\mathrm{C}-\mathrm{V}$ curves. Fig. 2 shows both the experimental and theoretical curves. The measured capacitance shows two distinct regions: a well defined peak in the voltage range from -0.8 to $0.2 \mathrm{~V}$ and a plateau like dependence from 0.5 to $1.5 \mathrm{~V}$. This second region will be discussed later. The behavior of the capacitance in the first range can be explained as follows: starting at $\mathrm{V}=-0.8 \mathrm{~V}$, the measured capacitance increases due to the filling of the dots, showing a peak at $\mathrm{V}=-0.11 \mathrm{~V}$ (the peak is broad due to fluctuations in the dots sizes); if the voltage in- creases, the capacitance signal diminishes because the dots are completely filled and for a further increase of the applied voltage, the total capacitance is given by the contribution of the bulk GaAs. From eq. (6), where it was used a gaussian function to describe the density of states, the peak observed in the $\mathrm{C}-\mathrm{V}$ curves is an evidence of an electron system characterized by a dimensionality lower than two. Since we do not expect the presence of an one-dimensional system, it seems clear that the negative differential capacitance in the -0.8 to $0.2 \mathrm{~V}$ voltage range confirms the presence of quantum dots, thus revealing its zero-dimensional character. Eq. (4) was numerically solved in the low temperature limit (8) using $\varepsilon_{S A Q D s}$ and $\Delta \varepsilon$ as fitting parameters. The fitting process was limited to the voltage range from the $1.0 \mathrm{~V}$ to $-3.0 \mathrm{~V}$, where the contribution of the dots dominates the capacitance of the structure. The model reproduces the experimental behavior and the fitted parameters of the zero-dimensional gaussian distribution were found to be $\varepsilon_{S A Q D s}^{C-V}=110 \mathrm{meV}$ and $\Delta \varepsilon^{C-V}=94$ meV (Fig. 2).

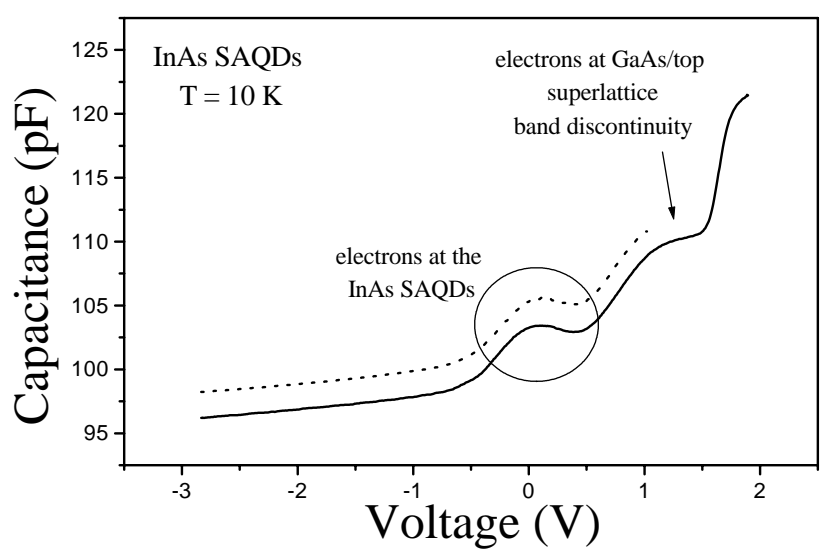

Figure 2. Experimental (solid line) and calculated (dotted line) capacitance-voltage curves for the sample with InAs SAQDs. The negative differential capacitance between -0.8 and $0.2 \mathrm{~V}$ is an evidence of the zero-dimensional character of the quantum dots.

As it is well established, the magnitude of the energy dispersion of electron states is directly related to the mean width of the photoluminescence (PL) spec- 
trum [10]. Fig. 3(a) shows the PL measurements taken at $10 \mathrm{~K}$. We only observe the luminescence lines related with the electron-hole recombination in the SAQDs $(E=1.24 \mathrm{eV})$ and in the GaAs $(E=1.51$ $e V)$. The energy $E=1.24 \mathrm{eV}$ with an energy broadening of $111 \mathrm{meV}$ (obtained by a gaussian fit), corresponds to an electron level located at $90 \mathrm{meV}$ below the conduction band of GaAs. It was calculated using the discontinuity energy for the conduction band $\Delta E_{C B}=0.85 \times\left(E_{G a A s}-E_{I n A s}\right)$. The other peak at $E=1.51 \mathrm{eV}$ is associated with the luminescence of the bulk GaAs. The excitation energy $\left(\varepsilon_{S A Q D s}\right)$ and the width $(\Delta \varepsilon)$ of the PL line associated with the SAQDs were found in accordance with the capacitance measurements $\left(\varepsilon_{S A Q D s}^{P L}=90 \mathrm{meV}\right.$ and $\left.\Delta \varepsilon^{P L}=111 \mathrm{meV}\right)$. This is a validation of the proposed model for the calculation of the capacitance-voltage curves.

Due to the sample construction geometry, the electrons can also be localized forming a $2 \mathrm{DEG}$ in both $(G a A s)_{4} /(A l A s)_{11} / G a A s$ top interface (indicated in Fig. 1) and in the wetting layer. In fact, we observed the presence of this kind of electron confinement on the measured capacitance as evidenced by the increase of the capacitance and the plateau observed from 0.5 $V$ to $1.5 V$ in Fig. 2. This observation is in accordance with the well defined relationship between capacitance and density of states: a two dimensional electron gas (2DEG) presents a step-like density of states which should produce a plateau-like dependence for the capacitance-voltage curves [12].

Some experimental facts allowed us to conclude that the observed plateau should be related to the confined electrons at the $(G a A s)_{4} /(A l A s)_{11} / G a A s$ top interface: the PL measurements plotted in Fig. 3(a) did not show evidences of the contribution of the wetting-layer which was confirmed by additional Raman scattering measurements, as we recently reported [13]. Also, there is no evidence of the excited states of the SAQDs in both the PL spectrum and $\mathrm{C}-\mathrm{V}$ measurements. By applying a forward bias to the sample, a 2DEG at the $(G a A s)_{4} /(A l A s)_{11} / G a A s$ top interface is formed and a plateau is observed in the $\mathrm{C}-\mathrm{V}$ curves. At a high enough voltage the 2DEG is depleted of electrons and a decrease of the capacitance is observed in the $\mathrm{C}-\mathrm{V}$ curves (Fig. 2).

An additional confirmation of the origin of the plateau in the measured capacitance can be obtained from the calculation of the spatial distribution of the charges in this sample calculating the $\mathrm{C}-\mathrm{V}$ profile $[14,15]$,

$$
N_{C-V}(z)=\frac{-2}{q S^{2} \varepsilon_{s}}\left[\frac{d C^{-2}}{d V}\right]^{-1}
$$

and

$$
z=S \frac{\varepsilon_{s}}{C}
$$

where $N_{C-V}$ is the density of electrons at $z$. A very sharp peak in the electron density was obtained when using the eq. (9) to calculate the profile [Fig. 3(b)]. This peak at $z=110 \mathrm{~nm}$ reflects the electron accumulation at the GaAs/top superlattice as expected from the analysis of the $\mathrm{C}-\mathrm{V}$ curves.
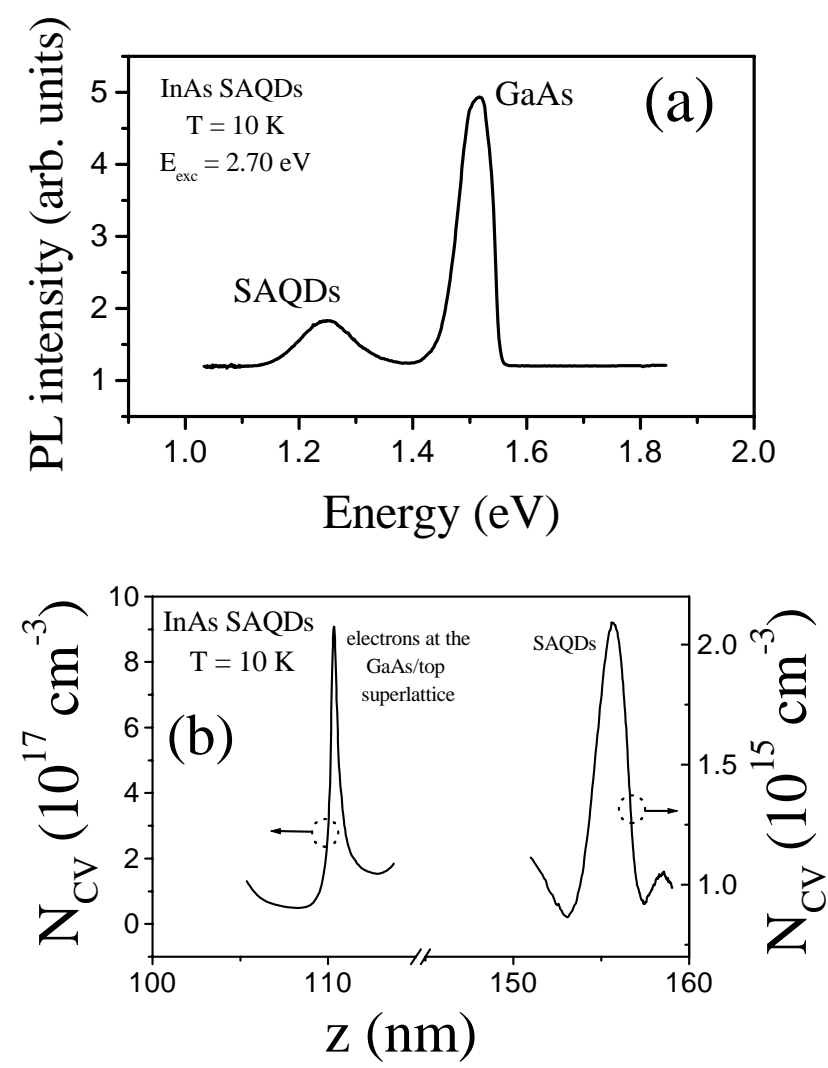

Figure 3. (a) PL spectrum of the sample with InAs SAQDs at $\mathrm{T}=10 \mathrm{~K}$. The lines at $\mathrm{E}=1.24 \mathrm{eV}$ and $\mathrm{E}=1.54 \mathrm{eV}$ are related to the SAQDs and GaAs bulk luminescences, respectively. Panel (b) shows the electron ditribution along the growth direction. It was calculated using the capacitancevoltage data.

In the voltage range where the negative differential capacitance is dominant, the conventional equations [eq. (9) and eq. (10)] for the C-V calculation are not valid and thus we use an alternative method as described in our previous work [5]. In the following some relevant aspects of this new method are given. An alternative and direct way for the construction of the $\mathrm{C}-\mathrm{V}$ profile in our samples is the calculation of a general expression for the spatial electronic distribution along the structure as a function of the total capacitance:

$$
N_{C-V}(w)=F(C) \equiv F(w)
$$

with

$$
C=G\left(C_{B u l k}, C_{S A Q D s}\right)
$$

where $w$ is the depletion region, $F, G$ are unknown functions. The formal dependence established by the 
eq. (11) is only possible to obtain if there is a well defined relation between the capacitance and the depletion width. In a quantum dots system, this relation cannot be determined because the depletion approximation is not valid for a system with dimensionality lower than 3 [the eqs. (9) and (10) were deduced in this approximation]. In other words, an expression which gives the relation between the electron distribution and the capacitance like eq. (9) is not possible to be achieved for a system containing quantum dots.

Describing the system (dots) as a pure electrostatic system embedded in a bulk structure, the dependence between the distribution of electrons and capacitance allowed us to determine the form of function $F$ as follows $[5]$ :

$$
N_{C V}=\frac{C_{B u l k}^{3}}{q \varepsilon_{s}}\left(\frac{d C_{B u l k}}{d V}\right)^{-1}\left[1+\frac{q t_{1}}{\varepsilon_{s}} \frac{\Delta n}{\Delta V}\right]
$$

with

$$
w=\frac{\varepsilon_{s}}{C_{B u l k}} S
$$

where $C_{B u l k}$ is the capacitance of the bulk structure without quantum dots. Notice that eq. (13) can be writen as

$$
N_{C V}=F_{1}\left(C_{B u l k}\right)+F_{2}\left(C_{S A Q D s}\right),
$$

where

$$
\begin{aligned}
F_{1}\left(C_{\text {Bulk }}\right) & =\frac{C_{\text {Bulk }}^{3}}{q \varepsilon_{s}}\left(\frac{d C_{B u l k}}{d V}\right)^{-1} \\
F_{2}\left(C_{S A Q D s}\right) & =\left[\frac{C_{B u l k}^{3}}{q \varepsilon_{s}}\left(\frac{d C_{B u l k}}{d V}\right)^{-1}\right] \times\left[\frac{q t_{1}}{\varepsilon_{s}} \frac{\Delta n}{\Delta V}\right]
\end{aligned}
$$

This means that the unknown dependence of the confined electrons upon the width of the depletion region is determined by the product indicated in function $F_{2}\left(C_{S A Q D s}\right)$ above. The dependence of the function $F_{2}\left(C_{S A Q D s}\right)$ on the nominal position of the dots plane $\left(t_{1}\right)$ give an uncertainty in the amplitude of the $\mathrm{C}-\mathrm{V}$ profile. It is worthwhile to notice two points: firstly, we consider a linear form for the unknown function $G$ which states the dependence of the total capacitance $\left[C=C_{B u l k}+C_{S A Q D s}\right.$, in eq. (12)] on the bulk and dots capacitance; secondly, the dots contribution in eq. (13) is proportional to the capacitance of the dots per unity of charge and area, and implicitly contains the dimensionality of the confined system through the density of states.

Using the model described above, the $\mathrm{C}-\mathrm{V}$ profile was calculated and the peak at $z=155 \mathrm{~nm}$ reflects the contribution of the electrons confined in the SAQDs
[Fig. 3(b)]. The density of electrons in the plane containing the dots can be obtained from the $\mathrm{C}-\mathrm{V}$ profile by the integration of the $N_{C-V} \times w$ curves [16]: an average value $n \simeq 5 \times 10^{9} \mathrm{~cm}^{-2}$ was found.

In summary, this paper presented a study of the capacitance-voltage characteristics in the InAs quantum dots system embedded in an undoped GaAs matrix. The proposed model reproduces essential features of the studied system and it was based on the analysis of the solution of the Poisson's equation and in the well defined relationship between capacitance and density of sates. As a result, the contributions of the electrons confined in zero- (SAQDs), bi- $\left[(G a A s)_{4} /(A l A s)_{11} / G a A s\right.$ interface $]$ and threedimensional (bulk) states were distinguished in the measured capacitance. In addition, the energy dispersion and the electron excitation energy of the SAQDs were obtained in close agreement with the photoluminescence measurements.

The authors would like to acknowledge E. Diagonel for his help with the sample preparation.The financial support of Brazilian agencies CNPq and FAPESP is also acknowledged.

\section{References}

[1] M. Grundmann, O. Stier, and D. Bimberg, Phys. Rev. B 52, 11969 (1995).

[2] R. C. Ashoori, H. L. Störmer, J. S. Weiner, L. N. Pfeiffer, S. J. Pearton, K. W. Baldwin, and K. West, Phys. Rev. Lett. 68, 3088 (1992).

[3] G. Medeiros-Ribeiro, D. Leonard, and P. M. Petroff, Appl. Phys. Lett. 66, 1767 (1995).

[4] S. Anand, N. Carlsson, M. E. Pistol, L. Samuelson, and W. Seifert, Appl. Phys. Lett. 67, 3016 (1995).

[5] A. J. Chiquito, Yu. A. Pusep, S. Mergulhão, J. C. Galzerani, and N. T. Moshegov, Phys. Rev. B 61, 5499 (2000).

[6] H. Drexler, D. Leonard, W. Hansen, J. P. Kotthaus, and P. M. Petroff, Phys. Rev. Lett. 73, 2252 (1994).

[7] T. P. Smith III, K. Y.. Lee, C. M. Knoeldler, J. M. Hong, and D. P. Kern, Phys. Rev. B 38, 2172 (1988).

[8] Ph. Lelong, O. Heller, G. Bastard, Physica E 2, 678 (1998).

[9] J. M. Lopez-Villegas, P. Roura, J. Bosch, J. R. Morante, A. Georgakilas, and K. Zekentes, J. Electrochem. Soc. 140,1492 (1993).

[10] P. N. Brounkov, A.A. Suvorova, N. A. Bert, A. R. Kovsh, A. E. Zhukov, A. Yu. Egorov, V. M. Ustinov, A. F. Tsatsulnikov, N. N. Ledentsov, P. S. Kopev, S. G. Konnikov, L. Eaves, and P. S. Main, Semiconductors 32, 1096 (1998).

[11] K. Hatori, J. Appl. Phys. 78, 4543 (1995).

[12] S. Luryi, Appl. Phys. Lett. 52, 501 (1988).

[13] Yu. A. Pusep, G. Zanelatto, S. W. Silva, J. C. Galzerani, P. P. Gonzalez-Borrero, A. I. Toropov, and P. Basmaji, Phys. Rev. B 58, R1770 (1998). 
[14] E. H. Rhoderick and R. H. Williams, Metalsemiconductor Contacts (Oxford, Cambridge), 1988.

[15] P. Blood, Semicond. Sci. Tech. 1, 1 (1986).
[16] E. F. Schubert, Semiconductors and Semimetals Vol.

40, (New York, Academic Press), 1994 